\title{
Dermatopathology: An abridged compendium of words. A discussion of them and opinions about them. Part 6 (I-L)
}

\author{
Bruce J. Hookerman ${ }^{1}$
}

1 Dermatology Specialists, Bridgeton, Missouri, USA

Citation: Hookerman BJ. Dermatopathology: An abridged compendium of words. A discussion of them and opinions about them. Part 6 (I-L). Dermatol Pract Concept. 2014;4(4):1. http://dx.doi.org/10.5826/dpc.0404a01

Copyright: @2014 Hookerman. This is an open-access article distributed under the terms of the Creative Commons Attribution License, which permits unrestricted use, distribution, and reproduction in any medium, provided the original author and source are credited.

Corresponding author: Bruce J. Hookerman, M.D., 12105 Bridgeton Square Drive, St. Louis, MO 63044, USA. Email: bjhookerman@aol.com

\section{$-\mathrm{I}-$}

ICHTHYOSIS: a generic term for skin conditions characterized by what are said to be fishlike scales, i.e., scales that are broad and polygonal with free edges, as are seen in ichthyosis vulgaris (and its look-alike, acquired ichthyosis), X-linked ichthyosis, and lamellar ichthyosis. Conditions reputed to be ichthyosis, such as ichthyosis hystrix and ichthyosis linearis circumflexa, do not qualify because they are not associated with broad polygonal scales.

ID: a suffix employed by dermatologists in such an inconsistent way that no unifying theme can be identified for it. For example, "-id" appears in words that reflect wholly diverse pathologic processes, such as eczematoid, syphilid, dermatophytid, monilioid, and leukemid. Sometimes, the suffix is used alone as in "id reaction," which has been invoked traditionally by dermatologists for what is known, equally confusingly, as "autoeczematization" and "autosensitization.” These fuzzy concepts were spawned more than three quarters of a century ago, when it was common practice in dermatology to use treatments such as autohemotherapy for a host of inflammatory diseases, injections intravenously of calcium gluconate for intractable pruritus, and radiation therapy for epilation of hairs harboring the organisms of tinea capitis. Just as those three modes of therapy have disappeared, so, too, should the concept of “-ids." We use the term "id reaction" only for a spongiotic dermatitis manifested by tiny vesicles on the hands of patients with florid dermatophytosis at another site, usually the feet, or for an analogue of that phenomenon such as widespread vesicles that appear subsequent to injudicious treatment, i.e., with Gentian violet (known sardonically in times past as "Gentian violent") of an exuberant spongiotic dermatitis, usually on the feet, such as an allergic contact dermatitis. A time-honored explanation for an "id" reaction is hematogenous dissemination of antigen, such as a component of a dermatophyte or a contact allergen (i.e., used to add explanatory information or to state something in different words).

IMMATURE SEBOCYTE: is a sebaceous cell with a round nucleus and scant cytoplasm devoid of vacuoles, such as, is present at the very periphery of a normal sebaceous gland and in proliferations with sebaceous differentiation, i.e., the benign sebaceoma and the malignant sebaceous carcinoma. In a normal sebaceous gland (SEE LOBULE), an immature sebocyte travels from the outermost boundary of a lobule to the very center of it where, nearby, resides a sebaceous duct. In the course of the journey, the cytoplasm enlarges greatly as more and more vacuoles laden with lipid come to bloat it, the nucleus becoming scalloped as a result of pressure on it by cytoplasm ever-expanding by virtue of the presence of ever more vacuoles filled increasingly with lipid. 
IMMUNOHISTOCHEMISTRY: denotes the study of tissues by analysis of their chemicals and, more specifically, by methods of immunology in which an antigen-antibody reaction is fundamental. For instance, immunoperoxidase is an immunohistochemical method in which determination of the chemical composition of a tissue uses an antigen-antibody reaction as a pivotal marker. Related methods are enzyme histochemistry, which employs an enzymatic reaction as the main process by which the chemical composition of a tissue is determinated, and radio labeling, which utilizes radioisotopes to that end. Immunohistochemistry is an adjunctive procedure to $\mathrm{H} \& \mathrm{E}$. It is meant to help as one other histopathologic "tool."

IMPETIGINIZATION: secondary infection of a lesion by bacteria, usually by virtue of animated scratching. Lesions that are impetiginized simulate impetigo clinically because of the presence of vesiculopustules or pustules, both of which eventuate in crusts. They resemble impetigo histopathologically because bacteria, often in clumps, are seen to adorn crusts and scale-crusts. Pruritic spongiotic dermatitides, such as allergic contact dermatitis and nummular dermatitis, often undergo impetiginization as a consequence of excoriation animatedly.

INCONTINENCE OF PIGMENT: loss of pigment from the epidermis that is due to damage of epidermal melanocytes and basal keratocytes and ingestion of the melanin by macrophages within the dermis. In some conditions, however, transfer of pigment to dermal macrophages may result from phagocytosis of the terminal portions of dendritic processes that have protruded through the basal lamina.

INDIAN-FILE ARRANGEMENT OF NEOPLASTIC CELLS: epithelial cells with atypical nuclei arranged as single cells (strands) between collagen bundles; especially common in metastatic carcinomas and in certain leukemias and can also be seen in melanoma among others.

INFERIOR SEGMENT OF A HAIR FOLLICLE: is bounded above by the site of insertion of muscle of hair erection above and below by the base of the follicle. This can be stated in more detail. In a longitudinal section, a mature follicle may be divided histologically into (1) an upper segment constituted of a single part, i.e., the isthmus which is delimited above by the entry of the sebaceous duct into the base of the infundibulum and below by desquamation of cornified cells of the inner sheath, and (2) a lower segment that consists of two parts, i.e., the stem, which stretches from the base of the isthmus to the end of the keratogenous zone at Adamson's fringe, and the bulb, which is the part of a follicle that resides below the Adamson's fringe. Adamson's fringe is the boundary between nucleated cells of a hair in the bulb of a follicle and enucleated cells of the hair in the stem of a follicle. The infundibulum is epidermal and not part of the hair follicle.
INFILTRATE: elements present in tissue in a quantity greater than normal. An infiltrate may be cellular or noncellular. When it is cellular, an infiltrate may be composed of inflammatory cells, i.e., lymphocytes, or neoplastic cells, i.e., those of metastatic carcinoma. When it is largely noncellular, an infiltrate may consist, for example, of mucin, amyloid, colloid, or urate. Noncellular infiltrates are referred to as deposits. An infiltrate made up of a single type of cell is considered to be monomorphous, examples of that being small lymphocytes in the inflammatory disease called polymorphous light eruption, abnormal lymphocytes in some lymphomas, and mast cells in the benign neoplasm urticaria pigmentosa. An infiltrate that is polymorphous, known also as mixed-cell, is composed of several types of cells that are present concurrently. For example, polymorphous infiltrates of neutrophils, eosinophils, lymphocytes, plasma cells, and histiocytes are seen in plaques of granuloma faciale/erythema elevatum diutinum. "Polymorphous" should not be confused with "pleomorphic," which refers to variation in size and shape of nuclei of neoplastic cells.

INFILTRATING MARGINS: an animistic term applied to the interposition of neoplastic cells between collagen bundles; intended to mean a poorly circumscribed (usually malignant) neoplasm. This cannot be seen under a microscope. It is similar to "invasion."

INFLAMMATION: changes in tissue that occur typically as a reaction to injury of any kind (i.e., mechanical, actinic, chemical, allergic, infectious, and effects of neoplasms) and characterized clinically be redness especially and swelling, and histopathologically by vasodilation and infiltrates of inflammatory cells, those especially being neutrophils, eosinophils, lymphocytes, plasma cells, and histiocytes.

INFLAMMATORY DERMATOSIS: a dermatosis is any pathologic condition of the skin. Dermatologists and dermatopathologists sometimes speak of "inflammatory dermatosis" when "dermatitis" is preferable. General pathologists do not allude to "acute appendicosis" or to "ulcerative colossi," but to appendicitis and colitis. The term "dermatitis" or the phrase "inflammatory disease of the skin" is preferable to "inflammatory dermatosis" because the word dermatosis is generic, not specific, and is employed by some authors for conditions other than inflammatory ones.

INFLAMMATORY DISEASE: a disorder characterized histopathologically by an infiltrate of inflammatory cells and, at times, by changes secondary to the effects of them such as spongiosis, acanthosis, and fibroplasia, but that infiltrate being unaffiliated with cells of any other fundamental pathologic process, (i.e., neoplastic, hamartomatous, cystic, etc.)

INFUNDIBULAR TUNNELS: are dilated, tortuous channels of infundibular epithelium filled with cornified cells usually 
in laminated array, within the substance of some seborrheic keratosis. They are known also and inaccurately as "horn pseudocysts" but they are not true cysts because they communicate with the skin surface through patulous ostia.

INFUNDIBULUM OF THE EPIDERMIS: an infundibulum, as its name denotes, has the shape of a funnel. The upper two-thirds of it consists of the cone of the funnel, whereas the lower one-third-sometimes referred to inaccurately as the infra-infundibulum-is formed by the narrow tube of funnel. The infundibulum is not part of the outer sheath, but is integral to epidermis; histologically, it is not epidermoid, as often is said, but epidermal. Although the epithelium of the lower tubular part of an infundibulum differs from that of the upper cone-like portion by having walls parallel to one another, slightly thinner granular zone, and fewer corneocytes, for practical purposes, infundibular epidermis is identical morphologically to surface epidermis, with which it is continuous. The length of infundibula varies greatly on different anatomic sites, being particularly long and dwarfing vellus follicles on a face, and being much shorter on a leg, for example. On one anatomic site, infundibula are unaffiliated with a follicle, namely, the nipple where a lactiferous (apocrine) duct enters directly into infundibula.

INNER SHEATH or INNER ROOT SHEATH: of a hair follicle is the part that actually encloses a hair along its course until the isthmus is reached. It consists of three layers: Henle's layer, Huxley's layer, and cuticle. The inner sheath cornifies in a distinctive fashion with formation first of bright red trichohyalin granules that disappear in time, a prelude to emergence of compactly arranged blue-gray corneocytes. The inner sheath comes into being through differentiation of matrical cells (one of seven separate paths of its differentiation).

IN SITU: is a term used to denote neoplasms that are confined to their site of origin. This usage presumes in speaking of malignant neoplasms that, untreated, the process could eventuate in metastatic carcinoma. This prediction of biologic behavior by a histopathologist is based on retrospective analysis of similar lesions. Cells of a malignant neoplasm that are confined to epithelium need not eventuate in metastases; they may remain in situ for the life of a person, and even regress. Unfortunately, a histopathologist cannot predict the behavior of those cells and, for that reason, malignancy in situ must be removed completely to insure that it will never progress to the point that it may prove fatal.

The concept of in-situ malignancy of the skin is accepted for neoplasms with squamous differentiation, i.e., Bowen's disease (see below), and for glandular differentiation, i.e., extramammary Paget's disease. Superficial basal-cell carcinoma is seated beneath the epidermis and therefore is not considered to be truly in situ, i.e., intra-epidermal. Melanoma in situ is a proliferation of abnormal melanocytes, confined to the epidermis and epithelial structures of adnexa, that fulfills all of the intra-epithelial criteria for melanoma. Virtually all primary cutaneous melanomas begin within the epidermis, i.e., in situ.

Parenthetically, the above contains two egregiously flawed concepts; the concept of "in situ" squamous cell carcinoma and also "invasive" squamous cell carcinoma and these should be jettisoned. Unlike the situation for apocrine carcinoma in situ (extra mammary Paget's disease) and melanoma in situ in which neoplastic apocrine cells and neoplastic melanocytes, respectively, actually move in vivo from the epidermis into the dermis and can be identified morphologically for what they are at both sites, that is not the case for so called squamous cell carcinoma in situ; neoplastic keratocytes do not descend as individual units or as discrete aggregations from the epidermis into the dermis, but rather the epidermis made up of abnormal keratocytes advances as a phalanx ever deeper. That being so, no normal epidermis is residual in a lesion of Bowen's disease (or solar keratosis), unlike the situation in extramammary Paget's disease which begins as apocrine carcinoma in situ and in melanoma in situ and furthermore, no clear distinction can be made by conventional microscopy between so called in situ squamous cell carcinoma and so called invasive squamous cell carcinoma; it is continuum, in contradistinction to the situation in extramammary Paget's disease and in melanoma where, at a moment in time, a plain distinction can be made between the component of the malignant neoplasm in the epidermis and the component of that same neoplasm in the papillary dermis.

A discussion of why this "phalanx" occurs follows. When neoplastic cells other than keratocytes are present within the epidermis and its adnexal epithelium, those cells often are separated from their surroundings by clefts. In apocrine carcinoma in-situ and melanoma in-situ spaces between neoplastic cells and adjacent keratocytes are encountered commonly. The clefts represent artifacts that come into being during the process of fixation of specimens as a consequence of poor attachment of neoplastic cells that are not keratocytes to contiguous normal epidermal keratocytes. In contrast, the neoplastic cells of what is squamous cell carcinoma in-situ, being the keratocytes that they are, are linked to seemingly unaffected keratocytes at the circumference of the neoplasm, and even within it, as well as to one another, by intercellular bridges. Moreover, the epidermis of "squamous cell carcinoma in-situ" tends to be largely replaced by neoplastic keratocytes, those cells usually making up the bulk of the effected surface epithelium. The situation for apocrine carcinoma in-situ and melanoma in-situ is different from that of "squamous cell carcinoma in-situ"; keratocytes often predominate over neoplastic nonkeratocytes in the affected surface epithelium of those incipient malignant neoplasms. 
It is rather easy to distinguish neoplastic apocrine cells and melanocytes from normal keratocytes within the epidermis, and that is also true after the neoplastic cells of those conditions have left the epidermal compartment and come to reside within the dermis, the reason being that the epidermis is so sharply demarcated from the dermis. But the neoplastic keratocytes of "squamous cell carcinoma in-situ" do not advance from the epidermis into the dermis in the same manner as do neoplastic apocrine cells and melanocytes. The reason is that nearly the entire epidermis of "squamous cell carcinoma in-situ" is composed of abnormal keratocytes and those neoplastic cells cause the surface epithelium to thicken progressively; the neoplastic keratocytes remain in continuity with the surface (and/or adnexal) epithelium in which the pathologic process began. Neoplastic keratocytes of so called squamous cell carcinoma in-situ do not transverse the dermo-epidermal junction to enter the dermis in the fashion of neoplastic apocrine cells and melanocytes but rather are part of steadily advancing mass of epithelium. In fact, in Bowen's disease, the stereotypical expression of "squamous cell carcinoma in-situ" in skin, the usual undulate pattern formed by rete ridges and dermal papillae is preserved, albeit altered markedly; even when the carcinoma has become so thick that it fills the upper half of the dermis.

In sum, a clear distinction can be made between apocrine and melanoma in-situ on one hand and "squamous cell carcinoma in-situ" on the other. When neoplastic cells of apocrine carcinoma in-situ and melanoma in-situ enter the dermis, the intradermal component is readily perceived as being separate from both the intraepidermal component of it and from epidermal keratocytes. That is not the case for what is called squamous cell carcinoma in-situ; that malignant neoplasm, no matter how thick it becomes, remains continuous with the surface and/or adnexal epithelium within which it originated. If neoplastic keratocytes of squamous cell carcinoma in the dermis or subcutaneous fat actually detach from the carcinoma above it, a metastasis has then come into being.

Another misguided idea is embodied in the cliché said to characterize what is purported to be squamous cell carcinoma in-situ, (this is a flawed concept) namely, "full thickness atypia" of surface epithelium. Before there can be involvement of the entire thickness of surface epithelium by changes of squamous cell carcinoma, earlier there must be involvement of the epithelium in only part of the thickness, much of the thickness, or the entire thickness. That circumstance, i.e., partial involvement, is no less a squamous cell carcinoma than involvement of the epithelium completely. An analogous situation obtains for melanoma in-situ; abnormal melanocytes of that neoplastic process disposed as solitary units only and stationed at the dermoepidermal junction exclusively is no less melanoma in-situ than when the entire thickness of the epidermis, including the cornified layer, is riddled by abnormal melanocytes.

It seems that a "lot of time" is spent in differentiating solar (actinic) keratosis from the flawed concept of "squamous cell carcinoma in-situ" by this search for "full thickness atypia" when actually solar (actinic) keratosis in its course may involve the entire thickness of the epidermis. Most dermatopathologists popularly but incorrectly equate "squamous cell carcinoma in-situ" and "full thickness atypia" with Bowen's disease (including bowenoid papulosis, erythroplasia of Queyrat) and lack of full thickness atypia with solar (actinic) keratosis (including radiation keratosis, arsenical keratosis, actinic chelitis). Interestingly, what is wrongly called "squamous cell carcinoma in situ” as a synonym for Bowen's disease is very different clinically and histopathologically from actinic keratosis. Consider the following discussion:

Bowen's disease, referred to conventionally and synonymously as squamous cell carcinoma in-situ, and bowenoid papulosis are as much very superficial squamous cell carcinomas as solar keratosis, radiation keratosis, and arsenical keratosis. The same abnormal keratocytes make up each of those conditions, only the silhouette of solar keratosis and its two analogues, Bowen's disease and bowenoid papulosis, are different; the cytopathologic attributes of all of them are the same. Bowen's disease, as a rule, appears in middle age or adulthood as a reddish plaque covered by adherent scale. When Bowen's disease occurs on the glanus penis, it is called erythroplasia of Queyrat. Cytopathologically, the cells that constitute Bowen's disease are indistinguishable from those that make up solar keratosis, but the architectural pattern of Bowen's disease, as assessed at low magnification of a conventional microscope, is different from that of solar keratosis. In Bowen's disease, the abnormal keratocytes form rectangles whereas in solar keratosis they form nubbins. In Bowen's disease, part of the thickness, much of the thickness, or the entire thickness of an epidermis that usually is thickened displays findings of squamous cell carcinoma, in contrast to solar keratosis in which only the lower part of the epidermis, that is, the nubbins, is affected in most instances but this may not be the case as noted earlier. Moreover, in Bowen's disease, never are there suprabasal clefts that contain acantholytic, dyskeratotic cells whereas in solar keratosis that distinctive pattern is common. It is not surprising; therefore, that "pseudoglandular" squamous cell carcinoma in the skin represents an advanced stage in the progression of solar keratosis but never of Bowen's disease.

INTEGUMENT: is synonymous with the entire skin. Including both hair-bearing and hairless parts.

INTERCELLULAR: means between adjacent cells. It often is used in the phrases "intercellular bridges," a synonym for 
desmosomes, and "intercellular edema," which is synonymous with spongiosis. Intercellular bridges, or spines, are seen to traverse intercellular spaces of epidermis and epithelial structures of adnexa in sections of skin visualized by conventional light microscopy.

INTRADERMAL MELANOCYTIC NEVUS: a nevus in which nests or fascicles of melanocytes are positioned in the dermis wholly. The term instructs only about where the melanocytes of a particular nevus are stationed; it conveys nothing about the kind of nevus, i.e., Clark's, Spitz's, Reed's, Miescher's, or Unna's.

INTRADERMAL VERTICAL GROWTH PHASE: (SEE RADIAL GROWTH PHASE)

\section{INTRAEPIDERMAL GROWTH PHASE (LATERAL OR HORIZONTAL GROWTH PHASE: (SEE RADIAL GROWTH PHASE)}

INTRAVASATION: the entrance of foreign material into a blood vessel. This term is used by some, while others would use "intravascular."

INVASION: through a microscope, no rational judgment can be made about "invasion" of neoplastic cells despite the insistence of histopathologists on making assessments of it. In fact, all such determinations are predicated entirely on thinking in post hoc ergo propter hoc fashion; first a histopathologist decides whether a neoplasm, for example of melanocytes, is benign or malignant and, if benign and involving both the epidermis and dermis, i.e., "classic" Spitz's nevus, the pronouncement is "not invasive," but if the decision about the very same neoplasm changes to malignant, i.e., melanoma affecting both the epidermis and dermis, then the declaration becomes "invasive": the "noninvasive" neoplasm suddenly becomes "invasive." In the ultimate analysis, "noninvasive" and "invasive" are irrelevant to histopathological diagnosis; criteria for diagnoses turn on morphological findings in regard both to silhouette and to character of constituent cells and not at all to mystical and fictitious concepts such as “invasion." In addition, "invasion” cannot be seen through a microscope. No movement of any kind can be seen on "fixed" tissue specimens.

IRIS LESIONS: ring-shaped papules with a central purpuric punctum and a peripheral rim of dusky erythema, nearly specific for erythema multiforme. Named for Iris, the goddess of the rainbow in Greek mythology, for whom the iris of the eye also was named.

IRREGULARLY PSORIASIFORM: denotes elongated rete ridges of uneven lengths, but with preservation of the normal undulating pattern between rete ridges and dermal papillae, as is seen in lichen simplex chronicus. This is strictly a descriptive term.
ISTHMIC PORTION OF A HAIR FOLLICLE: the portion of a hair follicle that is a bridge between the infundibular epidermis above and the stem below, bounded by the entry of the duct of the sebaceous gland above and the site of desquamation of corneocytes of the inner sheath below. There is no inner sheath there, the disappearance if it being responsible for the very creation of it.

ISTHMUS: the isthmus is delimited by desquamation of corneocytes of the inner sheath below and the entrance of the sebaceous duct at the base of the infundibular epidermis above, Isthmus means a narrow strip that connects two larger masses and, in cutaneous histology, the term is applied aptly to that short narrow strip of a follicle that is continuous with the stem of the follicle below and with the infundibular epidermis above. The isthmus is distinctive morphologically, fashioned as it is of epithelial cells arranged in a pattern unique to normal skin, except for that in follicles during the involutional phase of their cycle (catagen) when an appearance indistinguishable from that of isthmic epithelium is assumed by them. That epithelium, both of the isthmus and a follicle well advanced in catagen, is characterized by (1) a basal layer, (2) a spinous zone that is not truly "spinous" because intercellular "spines" are barely detectable between cells replete with pink cytoplasm, (3) absence of a granular zone, and (4) a prominent, brightly eosinophilic cornified layer whose cells are arranged compact and the surface of which is decorated by corrugations. The isthmus, which is the uppermost part of the follicle, lacks an inner sheath, but is a conduit for a hair en route from its origin as matrical cells in a bulb to the ostium of the infundibular epidermis and beyond it. Bulges from the lower half of the isthmus and the uppermost part of the stem are protrusions of the follicle to which muscles of hair erection are tethered by a tendon of fibrous tissue. Another important anatomic boundary alluded to previously is that marked by the entry of a sebaceous duct; that site demarcates the isthmus from the infundibular epidermis above. The isthmus alone forms the permanent "upper" segment of the hair follicle, the infundibulum being part of the epidermis.

ISTHMUS-CATAGEN CYST: is a cyst that occurs nearly always on a scalp and is lined by epithelium just like that at the isthmus of a normal follicle and that of a follicle well advanced in catagen. The name "isthmus-catagen cyst" is in synchrony with a method for naming cysts according to the normal epithelial structure of adnexa that the lining of the cyst most closely resembles, i.e., infundibular cyst, apocrine gland cyst. Synonyms for isthmus-catagen cyst are pilar cyst and tricholemmal cyst. "Pilar" means "hair," yet these cysts are devoid of hair. Tricholemmal refers to the entire outer sheath as it courses from the isthmus above through the stem to the bulb below, yet the lining of these cysts resembles only 
a discrete part of the outer sheath, to wit, that at the isthmus and that at the base of a follicle advanced in catagen.$$
-\mathrm{J}-
$$

JENTIGO: refers to a combination of simple lentigo and junctional melanocytic nevus. Its use now is passé. Each entity can be named accordingly.

JIGSAW PUZZLE PATTERN: describes resemblance vaguely to a jigsaw puzzle completed, that by virtue of proximity close of aggregations of epithelial cells of a proliferation benign, as occurs in cylindroma, but also at times in trichoblastoma of the type large nodular and in sebaceoma.

JUNCTIONAL ACTIVITY: a term introduced in the 1940s by Arthur Allen, but that lacks cogency because it pertains to a dynamic which cannot be visualized through a microscope, namely, activity of cells positioned mostly at the dermoepidermal junction. Allen believed that those cells, which in his view eventually were to become ones of melanoma, derived from epidermal keratocytes, an idea that long have been discredited.

JUNCTIONAL MELANOCYTIC NEVUS: a melanocytic nevus in which nests or fascicles of melanocytes are confined to the epidermis, especially to the dermoepidermal junction but the -term communicates nothing about the particular kind of nevus, i.e., Clark's, “Spitz's," or “Reed's," only about where histologically aggregations of melanocytes are situated.

JUVENILE MELANOMA: the term introduced by Sophie Spitz for a melanoma in children that has come to be proven benign and now is known as “classic” Spitz's nevus. Spitz insisted in her publication seminal of 1948 that by melanoma she meant malignant melanoma and she reiterated that in publications subsequent, only to acknowledge benignancy of it 3 years prior to her death in 1956. Actually in this article (according to Ackerman et al. "Spitz's Nevus": Reassessment Critical, Revision Radical. New York: Ardor Scribendi, Ltd., 2007) Sophie Spitz was dealing with two different nevi, not just one. One was a "classic" Spitz's nevus and the second was a combined congenital nevus with Spitz's cells and a third was what she called "spindle cell tumor."$$
-\mathrm{K}-
$$

KARYOLYSIS: disappearance of nuclei from cells during the process of cell death. Karyolysis (ghosts of nuclei), karyorrhexis (fragmentation of nuclei), and pyknosis (shrinkage and darkening of nuclei) are the three cardinal signs of necrosis. Karyolysis may be observed whenever necrosis occurs in the skin, i.e., ghosts of nuclei of severely ballooned keratocytes in general and ghosts of multinucleate epithelial giant cells in lesions induced by herpes virus, as one example, in particular. Karyolysis also may be noted in neoplastic diseases, i.e., "shadow" cells of pilomatricoma. The "shadows" referred to in that later benign neoplasm of follicular matrix cells actually represent ghosts of nuclei of cornified cells that fail in their attempt to produce a normal hair. Karyolysis is thought to result from progressive dissolution of chromatin as a consequence of the hydrolytic action of the DNA of lysosomes.

KARYORRHEXIS: dot-like debris of nuclei, know also as nuclear "dust," one of a triad of pivotal signs in nuclei, cells that are necrotic, namely, karyorrhexis, karyolysis, and pyknosis of cells that are necrotic. A synonym for karyorrhexis of white blood cells is leukocytoclasis, as is seen in leukocytoclastic vasculitis and in a host of other conditions as unrelated to one another as Sweet's syndrome and bullous lupus erythematosus. Because apoptosis is characterized by karyorrhexis and pyknosis, it qualifies as a type of necrosis. Karyorrhexis of lymphocytes is common in the subcutaneous fat of lupus profundus and karyorrhexis of lymphocytes ingested subsequently by histiocytes is known as "tingible bodies," as occurs in some examples of lymphocytoma cutis. Karyorrhexis of neoplastic lymphocytes occurs in subcutaneous T-cell lymphoma, the macrophages that gobble up the debris being dubbed beanbag cells.

KERATIN: consists of a group of 25 to 30 water-soluble, cytoskeletal proteins that form filaments $10 \mathrm{~nm}$ (intermediate size) in diameter. These filaments are characterized by their molecular weights (range of 40 to $70 \mathrm{kd}$ ) and their composition, namely, two types of keratin, one relatively larger and more basic, and the other smaller and more acidic. Keratins although related to one another biologically, are different from each other immunologically. They are found in all cutaneous epithelia and in the cornified layer of the epidermis. Keratins account for about $85 \%$ of the cellular protein. Keratins also are present in eccrine glands, apocrine glands, and sebaceous glands and their respective ducts. Keratins are found, too, in the epithelium of hair follicles and nail units. Keratins in hair and nails are considered to be "hard," characterized as they are by lack of formation of keratohyaline granules and by higher content of sulfhydryl. All other keratins are considered to be "soft." Histologists in the past used the term "keratin layer" as a synonym for the cornified layer of the epidermis, a use that is imprecise because it is incomplete.

KERATOCYTE: an epithelial cell of epidermal surface and infundibular epithelium, including basal, spinous, granular, and cornified layers, and of neoplasms made up of spinous cells, such as the benign pale-cell acanthoma and the malignant squamous-cell carcinoma. In a generic sense, all cells that contain keratin are keratocytes, but in actuality, the term has 
a more circumscribed meaning. Conventionally, the cells are called keratinocytes, but that is as wrong semantically as melaninocytes. The Greek root is "kerato" denoting horn. However, despite the above "keratinocyte" is still used by most.

KERATOGENOUS ZONE: refers to the zone in the lower segment of a follicle between noncornified and fully cornified elements, whose upper border is where the roof of a bulb meets the base of a stem, a boundary known also an Adamson's fringe (A-fringe).

KERATOHYALINE: refers to granules of different sizes and shapes that appear purple in sections stained by hematoxylin and eosin, and that are situated in the granular zone of the surface epidermis; the infundibular epidermis, and the sebaceous duct. Products of keratohyaline granules are essential to normal cornification of those epithelial structures. An increased quantity of abnormal keratohyaline granules is found in a variety of diseases (i.e., in the infundibula of verrucae vulgaris, lichen planopilaris, and prurigo nodularis).

KERATOHYALINE GRANULES: are deeply basophilic large granules found within spinous cells as they proceed to complete cornification in surface and infundibular epidermis and sebaceous duct.

KERATOSIS: refers to non-inflammatory hyperplasias and benign or malignant proliferations typified clinically and histopathologically by hyperkeratosis, i.e., orthokeratosis, parakeratosis, or both. The stereotypical examples of keratoses are verruca vulgaris (hyperplasia), seborrheic keratosis (benign neoplasm), and solar keratosis (malignant neoplasm). A lichen planus-like keratosis is merely a seborrheic keratosis in the process of undergoing regression secondary to the effects upon it of a band-like infiltrate of lymphocytes.

KOEBNER PHENOMENON: known also as isomorphic phenomenon, the induction by various types of trauma of lesions that are indistinguishable from lesions of the same disease that developed ab initio (from the beginning). The event is particularly common in psoriasis and lichen planus. It is to be differentiated from autoinoculation in infectious diseases, such as occurs in verrucae vulgaris and molluscum contagiosum.$$
-\mathrm{L}-
$$

LABYRINTHEAN: is a pattern resembling the appearance of a labyrinth, i.e., a place constructed of intricate passageways and blind alleys, compels extremely in arrangement. A labyrinthean pattern is encountered sometimes in sebaceomas and rarely in trichoblastic (basal cell) carcinoma.

LAMELLAR FIBROPLASIA: collagen bundles aligned parallel to one another and seated just beneath nests of melanocytes lodged at the base of epidermal rete ridges that often are long and thin. The findings may be observed in association with any proliferation of melanocytes at the dermoepidermal junction of an epidermis that exhibits long, rete ridges, as is the situation in simple lentigines, in junctional and compound nevi of certain kinds, and in some melanomas. Clark and co-workers consistently contrasted "lamellar fibroplasia" with "concentric eosinophilic fibroplasia," but, in actuality, the two are merely different names for bundles of collagen continous in array lamellar.

LAMINA DENSA: synonym for basal lamina. When the interface between dermis and an epithelium is studied by electron microscopy, the findings are as follows: Within a basal keratocyte, tonofilaments are attached to a hemidesmosome that parallels the cytoplasmic leaflet of the plasma membrane. The lamina lucida, an electron-lucent zone, separates the lower border of a plasma membrane from the electron-dense basal lamina densa (basal lamina). Within the lamina lucida, a sub-basal dense plaque is situated beneath a hemidesmosome and is traversed by fine anchoring filaments that extend in perpendicular fashion from the plasma membrane to mesh with the lamina densa. Cross-banded anchoring fibrils and bundles of dermal microfibrils extend downward from the lamina densa, where they interweave with Type III collagen fibers in the papillary dermis.

LAMINATED ORTHOKERATOSIS: describes a distinctive arrangement of corneocytes in which thin plates lie parallel to one another. The configuration is met within the stratum corneum of conditions such as ichthyosis vulgaris and X-linked ichthyosis, thin plates of corneocytes in those conditions being disposed parallel to the skin surface, as well as to one another. Laminated orthokeratosis is found, too, in a type of epidermal cyst, i.e., an infundibular cyst, and in a "pseudocyst," i.e., an infundibular tunnel in a lesion of seborrheic keratosis. Infundibular hyperkeratosis is the sine qua non for diagnosis of keratosis pilaris and the variant of it, lichen spinulosus, and is observed in diseases encountered not uncommonly, such as pityriasis rubra pilaris, lichen planopilaris, and discoid lupus erythematosus, and in ones that are encountered only rarely, such as scurvy, phrynoderma, and "avitaminosis" secondary to severe alcoholism. Hyperkeratosis of the upper part of eccrine ducts occurs in diseases such as lichen sclerosis et atrophicus and in milia that form after reeepithelialization has occurred beneath some subepidermal blistering diseases, i.e., porphyria cutanea tarda and bullous pemphigoid. Most milia, however, represent tiny infundibular cysts. (SEE HYPERKERATOSIS, BASKET WOVEN ORTHOKERATOSIS, COMPACT ORTHOKERATOSIS)

LANGER'S LINES: lines along which surgical incisions gape or fall together; depending on whether they are made across or parallel; charted by Langer, who pierced the skin of cadavers with an awl. 
LANGUAGE: The method of human communication either spoken or written consisting of the use of words in a structured and conventional way.

LEUKOCYTOCLASIS: fragmentation of leukocytes, i.e., karyorrhexis, with formation of nuclear "dust." The word usually refers to nuclei of polymorphonuclear leukocytes that become shattered into dust like particles in a variety of circumstances. Nuclei of lymphocytes or of any other inflammatory or neoplastic cell also may undergo karyorrhexis. Nuclear "dust" of neutrophils is the sine qua non for diagnosis of leukocytoclastic vasculitis, but it may be noted in other conditions as different as dermatitis herpetiformis and pyoderma gangrenosum. The stereotypic situation for appearance of nuclear "dust" of lymphocytes is lupus profundus, in which patchy lymphoplasmacytic infiltrates in lobules of the subcutaneous fat are accompanied by nuclear debris of lymphocytes in variable amount. Tingible bodies in germinal centers of some expressions of lymphocytoma cutis represent nuclear "dust" of lymphocytes that has been ingested by macrophages. In short, if the meaning of the word "leukocytoclasis" is to be understood clearly, it must be modified always by "of neutrophils" or "of lymphocytes."

LEVEL OF INVASION: the depth of extension into the skin of a melanoma according to Clark and his associates. They have defined five levels of extension.

Level I. Melanocytes of melanoma confined to the epidermis. (Melanoma in situ)

Level II. Melanocytes of a melanoma in the papillary dermis, but not the reticular dermis.

Level III. Melanocytes of a melanoma at the interface between the papillary and reticular dermis, filling the papillary dermis but not yet within the reticular dermis.

Level IV: Melanocytes of a melanoma in the reticular dermis. Level V. Melanocytes of a melanoma in the subcutaneous fat.

Levels of invasion are approximations of thicknesses of melanomas, and because seriousness of prognosis tends to vary nearly directly with thickness, levels of invasion have some validity as prognostic indices. In practice, however, determination of level is often difficult, particularly in anatomic areas where it is difficult to discern the interface between papillary and reticular dermis. Moreover, there is poor agreement in assessment of levels of invasion by pathologists in examination of given melanomas. Measurement of thickness of a melanoma by means of an ocular micrometer is more precise and repeatable. It therefore has supplanted determination of levels as a guide to prognosis. Someday it to will probably "disappear." It is only a "number" and does not take into account the "host" response and many other factors that determine the prognosis.
LICHENIFICATION: thickening of the skin secondary to rubbing forcefully for many months or years. The condition is typified clinically by accentuation of normal skin markings, hyperpigmentation, and induration of variable extent, a constellation of findings designated lichen simplex chronicus. Histopathologically, the surface epidermis of lichenified skin is compactly orthokeratotic, hypergranulotic, and unevenly acanthotic in psoriasiform fashion. The infundibular epidermis and upper part of the eccrine duct within the dermis sometimes show evidences of proliferation in pseudocarcinomatous fashion, the papillary dermis being thickened markedly by coarse bundles of collagen arranged in vertical streaks aligned parallel to rete ridges and perpendicular to the skin surface. Another expression of lichenification, one seen particularly on the scalp, genitalia, and mucous membranes, is characterized by compact orthokeratosis and hypergranulosis so extensive that at first glance the epidermis resembles that of normal palm or sole. In that circumstances in which the epidermis, at first blush, looks just like that of normal volar skin, a clue to the correct diagnosis of lichen simplex chronicus is the presence of folliculosebaceous units ("hairy palm sign”). Parenthically, folliculosebaceous units are not found on volar skin except in bulldogs. (Ackerman et al,.Histologic Diagnosis of Inflammatory Skin Diseases, $3^{\text {rd }}$ ed. New York: Ardor Scribendi, Ltd., 2005.)

Other classic examples of lichenification besides plaques of lichen simplex chronicus are papules and nodules of prurigo nodularis, the former (lichen simplex chronicus) brought into being by rubbing the skin, usually with knuckles, to and fro, in contrast to the latter (prurigo nodularis), which develops consequent to rubbing for a long time and with the ball of a first finger, a particular discrete site. Should prurigo nodularis become eroded or ulcerated following scratching energetically, the condition then is named picker's nodule. Lichenification can be produced in skin that is normal clinically or it can be imposed on a variety of chronic pruritic dermatitides, such as allergic contact dermatitis or nummular dermatitis, and on neoplastic processes, such as, mycosis fungoides and leukemia cutis. All lesions of atopic dermatitis are the result of the effects of rubbing (lichenification) and scratching (erosions and ulcers); none of those lesions "erupt."

LICHENOID: clinically, flat-topped papules and histopathologically, a band like infiltrate, usually of lymphocytes mostly, in a papillary dermis thickened by it. Nearly always, lichenoid infiltrates of an inflammatory process consist of lymphocytes, as in lichen planus, lichenoid discoid lupus erythematosus, and lichenoid purpura of Gougerot and Blum. Lymphocytes of a neoplastic process, to wit, mycosis fungoides in the plaque stage of the disease, also tend to be arrayed in band like fashion. As a rule, lichenoid infiltrates of inflammatory diseases obscure the dermoepidermal inter- 
face where they are accompanied by vacuolar alternation and necrotic keratocytes, but that is not the case invariably, i.e., in some examples of the lichenoid purpura of Gougerot and Blum where the band like infiltrate of lymphocytes may not impinge on the epidermis. Lichenoid infiltrates may be diffuse, i.e., across the entire expanse of a lesion, as in lichen planus or lichenoid purpura, or focal (patchy), i.e., confined to one or more spots along the front of a lesion, as in lichen nitidus or in one expression of lichen striatus. Lichen nitidus exemplifies the focal nature of some lichenoid dermatitides and illustrates that a single lichenoid process may be made up mostly of lymphocytes (at an early stage) and mostly of epithelioid histiocytes (at a late stage), at one period the two types of inflammatory cells being present in equal measure, concurrently. A band like infiltrate of leukocytes in a thickened papillary dermis is responsible for the appearance clinically of a papule whose surface is flat, the prime example of that being the basic lesion of lichen planus. Not all diseases marked by flat-topped papules, however, exhibit band like infiltrates in the papillary dermis, as is the case in longstanding lesions of lichen sclerosis et atrophicus, lichen simplex chronicus, and lichen myxedematosus.

LIPOCHROME: is an insoluble pigment composed of polymers of lipids and phosolipids in association with protein. Histopathologically, lipochrome is a yellow-brown, finely granular, intracytoplasmic pigment. It represents the indigestible residues of autophagic vacuoles formed during long-standing injury to cells or aging of them, and forms as a result of damage by free radicals and by perodixation of polyunsaturated lipid. Lipochrome is synonymous with "lipofuscin," “carotid," and “wear and tear" pigment.

LOBULAR PANNICULITIS: an inflammatory process in the panniculus adiposis recognizable at scanning magnifi- cation of a conventional microscope, in which the infiltrate of inflammatory cells is situated mostly in the lobules rather than in the septa.

LOBULE: denotes a discrete unit of cells bounded by fibrous tissue. Lobules in the subcutaneous fat are rectangular, comprised nearly entirely of adipocytes, and bounded by fibrous septa (trabeculae). Lobules of sebaceous, eccrine, and apocrine units consist of glandular segments surrounded by distinctive stroma, i.e., (sebaceous lobule). The epithelial units and nonepithelial components of cutaneous adnexal epithelia form a recognizable morphologic and functional unit. The term "lobule" also is applied to each of many similar-appearing units that make up entire neoplasms considered to be multilobular, i.e., tubular adenoma and cylindroma.

LUMEN: designates a channel within a tubule or within an endothelium. In normal skin, there are three distinct types of lumen within adnexal epithelium, namely, those of eccrine glands and ducts, apocrine glands and ducts, and sebaceous ducts. Normal apocrine glands are recognizable by distinctive signs of apocrine secretion, eccrine glands by presence of pale and dark cells, and sebaceous ducts by a thin, crenulated cornified layer. Ducts of apocrine and eccrine glands cannot be distinguished from one another morphologically. The presence of lumina within an epithelial proliferation indicates that cells around them have differentiated to make (glandular) or to transport (ductal) secretory or excretory products. Most lumina in skin, however, are vascular channels lined by endothelial cells.

LYMPHOID FOLLICLES: collections of tightly packed lymphoid cells that form oval to spherical nodules. They are called primary follicles when they are composed of small lymphocytes and secondary follicles when germinal centers form after primary follicles have been stimulated by antigen. 